\title{
Expanded Cord Blood Stem Cells Mixed with Engineered Human Endothelial Cells AB-110
}

National Cancer Institute

\section{Source}

National Cancer Institute. Expanded Cord Blood Stem Cells Mixed with Engineered

Human Endothelial Cells AB-110. NCI Thesaurus. Code C150135.

A population of ex vivo expanded, CD34-positive-enriched hematopoietic progenitor cells (HPCS), which are derived from allogeneic human umbilical cord blood (UCB), and are cocultured and expanded with a proprietary preparation of human endothelial cells (ECs), which are genetically modified with an adenovirus (Ad) vector that includes the Ad E4 region that encodes the ORF1 (E4ORF1) gene, that can be used for transplantation. Upon transplantation of the expanded cord blood stem cells mixed with eng ineered human ECs AB-110, the UCB-derived cells can differentiate into a variety of cell types and promote blood cell recovery. Compared to bone marrow transplants, these HPCs demonstrate a decreased risk of causing graft-versus-host disease (GvHD), increase survival and enhance the potential for transplant and eng raftment in any given patient as there is no need for a matched donor. Compared to HPCs alone, inclusion of the ECs increases blood stem cell count, enhances engraftment potential and increases the chances for blood cell recovery, thereby further increasing the potential for a successful cord blood transplantation. Insertion of the Ad E4ORF1 gene enhances survival and replication of the ECs ex vivo, preserves the ECs' vascular functions and improves the ability of the ECs to secrete angiocrines, which promotes stem cell proliferation and increases engraftment. 